\title{
On first-order periodic boundary value problems and distributional Henstock-Kurzweil integrals
}

\author{
Wei Liu*, Tianqing An and Guoju Ye
}

\section{"Correspondence:}

liuw626@hhu.edu.cn

Department of Mathematics, Hohai

University, Nanjing, 210098,

P.R. China

\begin{abstract}
This paper is devoted to the study of existence and dependence of solutions of the first-order periodic boundary value problems involving the distributional Henstock-Kurzweil integral. The methods used are mainly the method of upper and lower solutions and a fixed point theorem.

Keywords: periodic boundary value problem; distributional Henstock-Kurzweil integral; distributional derivative; extremal solutions; upper and lower solutions; fixed point
\end{abstract}

\section{Introduction}

In this paper we study the existence of extremal solutions of the first-order periodic boundary value problem (PBVP for short)

$$
D x=f(t)+h(t, x), \quad x(0)=x(T),
$$

where $D x$ stands for the distributional derivative of $x \in C[0, T], 0<T<\infty, h:[0, T] \times$ $C[0, T] \rightarrow C[0, T]$ and $f$ is a distribution (generalized function). More precisely, we study the dependence of the extremal solutions of PBVP (1.1) on $f$ and $h$.

It is known that the notion of a distributional derivative is very general, including, for example, ordinary derivatives and approximate derivatives. The first-order PBVP of the form

$$
\frac{d x}{d t}=h(t, x), \quad x(0)=x(T)
$$

with ordinary derivatives $\frac{d x}{d t}$ and $h:[0, T] \times \mathbb{R}^{n} \rightarrow \mathbb{R}^{n}$ has been studied extensively in recent years; see, for example, [1-4]. In [5], by using the distributional derivative, PBVP (1.2) has been generalized to (1.1) in the case when $h$ is continuous with respect to $x$. Results about the existence of solutions and the topological structure of the solution set are given. In this paper, we study PBVP (1.1) in the case when $h$ is monotone with respect to $x$ and obtain some extended results.

The outline of this paper is as follows. In Section 2 we define the distributional HenstockKurzweil integral or briefly the $D_{H K}$-integral. We say that a distribution $f$ is $D_{H K}$-integrable

○2014 Liu et al.; licensee Springer. This is an Open Access article distributed under the terms of the Creative Commons Attribution License (http://creativecommons.org/licenses/by/2.0), which permits unrestricted use, distribution, and reproduction in any medium, provided the original work is properly cited. 
on $[a, b] \subset \mathbb{R}$ if there is a unique continuous function $F$ on $[a, b]$ with $F(a)=0$ whose distributional derivative is $f$. From the definition of the $D_{H K}$-integral, we know that the $D_{H K}$-integral includes the Riemann, Lebesgue, Henstock-Kurzweil (briefly $H K$ ), and wide Denjoy integrals (for details, see [6-11]). Furthermore, the space of such integrable distributions is a Banach space and has many basic properties; see [5, 12].

In Section 3, by using the $D_{H K}$-integral and the distributional derivative, we generalize PBVP (1.2) to (1.1). Our main tools are the method of upper and lower solutions and a fixed point theorem. The main result is Theorem 3.1, which extends some corresponding results in $[1,2]$. This section also contains an illustration of the results.

\section{The distributional Henstock-Kurzweil integral}

In this section, we present the definition and some basic properties of the distributional Henstock-Kurzweil integral.

Define the space

$$
C_{c}^{\infty}=\left\{\phi: \mathbb{R} \rightarrow \mathbb{R} \mid \phi \in C^{\infty} \text { and } \phi \text { has compact support in } \mathbb{R}\right\},
$$

where the support of a function $\phi$ is the closure of the set on which $\phi$ does not vanish, denoted by $\operatorname{supp}(\phi)$. A sequence $\left\{\phi_{n}\right\} \subset C_{c}^{\infty}$ converges to $\phi \in C_{c}^{\infty}$ if there is a compact set $K$ such that all $\phi_{n}$ have supports in $K$ and for every $m \in \mathbb{N}$ the sequence of $m$ th derivatives $\phi_{n}^{(m)}$ converges to $\phi^{(m)}$ uniformly on $K$. Let $C_{c}^{\infty}$ be endowed with this convergence property and denote it by $\mathcal{D}$. Also, $\phi$ is called test function if $\phi \in \mathcal{D}$. Distributions are defined to be continuous linear functionals on $\mathcal{D}$. The space of distributions is denoted by $\mathcal{D}^{\prime}$, which is the dual space of $\mathcal{D}$. That is, if $f \in \mathcal{D}^{\prime}$ then $f: \mathcal{D} \rightarrow \mathbb{R}$ is a linear functional and we write $\langle f, \phi\rangle \in \mathbb{R}$ for $\phi \in \mathcal{D}$.

For all $f \in \mathcal{D}^{\prime}$, we recall that the distributional derivative $D f$ of $f$ is a distribution satisfying $\langle D f, \phi\rangle=-\left\langle f, \phi^{\prime}\right\rangle$, where $\phi$ is a test function and $\phi^{\prime}$ is the ordinary derivative of $\phi$. With this definition, all distributions have derivatives of all orders and each derivative is a distribution.

Let $(a, b)$ be an open interval in $\mathbb{R}$. We define

$$
\mathcal{D}(a, b)=\left\{\phi: \mathbb{R} \rightarrow \mathbb{R} \mid \phi \in C^{\infty} \text { and } \phi \text { has compact support in }(a, b)\right\} .
$$

Of course, $\mathcal{D}(a, b)$ is endowed with the above convergence property. The dual space of $\mathcal{D}(a, b)$ is denoted by $\mathcal{D}^{\prime}(a, b)$. In this case, if $a=-\infty, b=+\infty$, then $\mathcal{D}(a, b)=\mathcal{D}$ and $\mathcal{D}^{\prime}(a, b)=\mathcal{D}^{\prime}$.

Define $C[a, b]$ to be the space of continuous functions on $[a, b]$, and

$$
B_{C}=\{F \in C[a, b] \mid F(a)=0\} .
$$

Note that $B_{C}$ is a Banach space with the uniform norm $\|F\|_{\infty}=\max _{[a, b]}|F|$.

We are now able to present the definition of the $D_{H K}$-integral.

Definition 2.1 ([5, Definition 2.1]) A distribution $f$ is distributionally Henstock-Kurzweil integrable or briefly $D_{H K}$-integrable on $[a, b]$ if $f$ is the distributional derivative of a continuous function $F \in B_{C}$. 
The space of $D_{H K}$-integrable distributions is defined by

$$
D_{H K}=\left\{f \in \mathcal{D}^{\prime}(a, b) \mid f=D F \text { for some } F \in B_{C}\right\} .
$$

With this definition, if $f \in D_{H K}$ then, for all $\phi \in \mathcal{D}(a, b)$,

$$
\langle f, \phi\rangle=\langle D F, \phi\rangle=-\left\langle F, \phi^{\prime}\right\rangle=-\int_{a}^{b} F \phi^{\prime} .
$$

The second equality holds because $F$ and $\phi$ are continuous on $(a, b)$ and $\phi^{\prime}$ has compact support in $(a, b)$. The integral in the last equality exists as a Riemann integral for the same reason.

For convenience, we write $\left(D_{H K}\right) \int_{a}^{b} f=F(b)$, where $F$ is called the primitive of $f$ and ' $\left(D_{H K}\right) \int$ ' denotes the $D_{H K}$-integral. As usual, if $F \in C[a, b]$ and $\left(D_{H K}\right) \int_{a}^{b} f=F(b)-F(a)$, then the function $F$ is a primitive of $f$. Notice that if $f \in D_{H K}$ then $f$ has many primitives in $C[a, b]$, all differing by a constant, but $f$ has exactly one primitive in $B_{C}$.

Remark 2.1 Integrals defined in the same way have also been proposed in other papers. For example, Ang et al. [13] defined it in the plane and called it the G-integral, and Talvila [9] defined the $A_{C}$-integral on the extended real line. In that case of integration over onedimensional interval, these two integrals coincide.

The following result is known as the fundamental theorem of calculus.

Lemma 2.1 ([9, Theorem 4$])$

(a) Let $f \in D_{H K}$ and $F(t)=\left(D_{H K}\right) \int_{a}^{t} f$. Then $F \in B_{C}$ and $D F=f$.

(b) Let $F \in C[a, b]$. Then $\left(D_{H K}\right) \int_{a}^{t} D F=F(t)-F(a)$ for all $t \in[a, b]$.

For $u, v \in C[a, b]$, we say that $u \leq v$ if and only if $u(t) \leq v(t)$ for all $t \in[a, b]$. Similarly, for $f, g \in D_{H K}$, we say that $f \preceq g$ if and only if

$$
\left(D_{H K}\right) \int_{I} f \leq\left(D_{H K}\right) \int_{I} g \quad \text { for all } I \subset[a, b] .
$$

The following lemma will be needed later.

Lemma 2.2 ([13, Corollary 1]) If $f_{1}, f_{2}, f_{3} \in \mathcal{D}^{\prime}(a, b), f_{1} \preceq f_{2} \preceq f_{3}$, and if $f_{1}$ and $f_{3}$ are $D_{H K}$ integrable, then $f_{2}$ is also $D_{H K}$-integrable.

Let $f \in D_{H K}, F \in B_{C}$ with $D F=f$. Then, under the Alexiewicz norm

$$
\|f\|=\|F\|_{\infty}=\max _{[a, b]}|F|
$$

$D_{H K}$ is a Banach space (see [9, Theorem 2]).

We say that a sequence $\left\{f_{n}\right\} \subset D_{H K}$ converges strongly to $f \in D_{H K}$ (or $f_{n} \rightarrow f$ in $D_{H K}$ ) if $\left\|f_{n}-f\right\| \rightarrow 0$ as $n \rightarrow \infty$. The following two convergence theorems hold.

Lemma 2.3 ([13, Corollary 4, monotone convergence theorem $]$ ) Let $\left\{f_{n}\right\}_{n=0}^{\infty}$ be a sequence in $D_{H K}$ such that $f_{0} \preceq f_{1} \preceq \cdots \preceq f_{n} \preceq \cdots$, and that $A=\lim _{n \rightarrow \infty}\left(D_{H K}\right) \int_{a}^{b} f_{n}$. Then $f_{n} \rightarrow f$ in $D_{H K}$ and $\left(D_{H K}\right) \int_{a}^{b} f=A$. 
Lemma 2.4 ([13, Corollary 5, dominated convergence theorem $]$ ) Let $\left\{f_{n}\right\}_{n=0}^{\infty}$ be a sequence in $D_{H K}$ such that $f_{n} \rightarrow f$ in $\mathcal{D}^{\prime}$. Suppose that there exist $f_{-}, f_{+} \in D_{H K}$ satisfying $f_{-} \preceq f_{n} \preceq f_{+}$, $\forall n \in \mathbb{N}$. Then $f \in D_{H K}$ and $\lim _{n \rightarrow \infty}\left(D_{H K}\right) \int_{a}^{b} f_{n}=\left(D_{H K}\right) \int_{a}^{b} f$.

If $g:[a, b] \rightarrow \mathbb{R}$, its variation is $V g=\sup \sum_{n}\left|g\left(t_{n}\right)-g\left(s_{n}\right)\right|$, where the supremum is taken over every sequence $\left\{\left(t_{n}, s_{n}\right)\right\}$ of disjoint intervals in $[a, b]$. If $V g<\infty$ then $g$ is called a function with bounded variation. Denote the set of functions with bounded variation by $\mathcal{B V}$. As it is known that the dual space of $D_{H K}$ is $\mathcal{B V}$ (see details in [9]), we have the next result.

Lemma 2.5 ([9, Definition 6, integration by parts]) Let $f \in D_{H K}$ and $g \in \mathcal{B V}$. Define $f g=$ $D H$, where $H(t)=F(t) g(t)-\int_{a}^{t} F d g$. Then $f g \in D_{H K}$ and

$$
\left(D_{H K}\right) \int_{a}^{b} f g=F(b) g(b)-\left(D_{H K}\right) \int_{a}^{b} F d g .
$$

Denote by $L$ the space of Lebesgue integrable functions and by $\|\cdot\|_{L}$ the norm on $L$.

Definition 2.2 Let $f \in D_{H K}$ and $g \in L$. Let $\left\{g_{n}\right\} \subset \mathcal{B V}$ such that $\left\|g_{n}-g\right\|_{L} \rightarrow 0$. Define $f g$ as the unique element in $D_{H K}$ such that $\left\|f g_{n}-f g\right\| \rightarrow 0$.

This definition, which is modified from [10, Definition 5], makes sense since $\mathcal{B V}$ is dense in $L$. Moreover, the next statement holds.

Lemma 2.6 ([5, Lemma 2.7]) Let $f$, $g$ be the distributional derivatives of $F, G$, respectively, where $F, G \in C[a, b]$. Then

$$
D(F G)=f G+F g .
$$

\section{Periodic boundary value problems}

In this section, we shall study the first-order periodic boundary value problem

$$
D x=f(t)+h(t, x), \quad x(0)=x(T),
$$

where $D x$ denotes the distributional derivative of $x \in C[0, T], h:[0, T] \times C[0, T] \rightarrow$ $C[0, T]$ and $f$ is a distribution on $[0, T]$. Throughout this section, we denote by $D_{H K}$ (respectively, $H K, L)$ the space of $D_{H K}$ (respectively, $H K$, Lebesgue)-integrable functions and by ' $(*) \int$ ' the $*$-integral.

For convenience, let us list the following assumptions on the functions $f$ and $h$.

$\left(D_{1}\right)$ There exist $y, z \in C[0, T], c_{y}, c_{z} \in D_{H K}$ such that $y \leq z$ and

$$
D y \preceq f+h(\cdot, y)-c_{y} \quad \text { and } \quad D z \succeq f+h(\cdot, z)+c_{z} \quad \text { on }[0, T] \text {, }
$$

and there is $p \in H K$ such that $p \geq 0$ on $[0, T], P(T) \neq 0$ holds for the function

$$
P(t)=(H K) \int_{0}^{t} p(s) d s
$$


and the inequalities

$$
y(0)-y(T) \leq\left(D_{H K}\right) \int_{t}^{T} e^{P(s)-P(T)} c_{y}(s) d s+\left(D_{H K}\right) \int_{0}^{t} e^{P(s)} c_{y}(s) d s
$$

and

$$
z(T)-z(0) \leq\left(D_{H K}\right) \int_{t}^{T} e^{P(s)-P(T)} c_{z}(s) d s+\left(D_{H K}\right) \int_{0}^{t} e^{P(s)} c_{z}(s) d s
$$

are true for $t \in[0, T]$.

$\left(D_{2}\right) h(\cdot, x(\cdot))$ is Lebesgue integrable for every fixed $x \in[y, z]$, and the distribution $f$ is $D_{H K^{-}}$ integrable on $[0, T]$.

$\left(\mathrm{D}_{3}\right) h(t, x)+p(t) x$ is nondecreasing in $x \in[y, z]$ for all $t \in[0, T]$.

We recall that $[y, z]:=\{x \in C[0, T] \mid y(t) \leq x(t) \leq z(t)$ for all $t \in[0, T]\}$.

Before coming to the main results in this paper, we give a result following from Lemma 2.1, that is, PBVP (1.1) can be converted to an integral equation.

Lemma 3.1 Let $f$ and h satisfy $\left(D_{2}\right)$. A function $x:[0, T] \rightarrow \mathbb{R}$ is a solution of PBVP (1.1) on $[0, T]$ if and only if the equality

$$
\begin{aligned}
x(t)= & e^{-\hat{P}(t)}\left(D_{H K}\right) \int_{0}^{t} e^{\hat{P}(s)}(f(s)+h(s, x(s))+\hat{p}(s) x(s)) d s \\
& +\frac{e^{-\hat{P}(t)}}{e^{\hat{P}(T)}-1}\left(D_{H K}\right) \int_{0}^{T} e^{\hat{P}(s)}(f(s)+h(s, x(s))+\hat{p}(s) x(s)) d s
\end{aligned}
$$

is true for any $\hat{p} \in H K$ such that $\hat{p} \geq 0$ on $[0, T]$ and

$$
(H K) \int_{0}^{T} \hat{p}(s) d s \neq 0
$$

Remark 3.1 In view of Lemma 2.5, the result $e^{\hat{P}(t)} \in \mathcal{B V}$ on $[0, T]$ implies that $e^{\hat{P}(t)}(f(t)+$ $h(s, x(s))+\hat{p}(s) x(s))$ is $D_{H K}$-integrable on $[0, T]$, because $f(t)+h(s, x(s))+\hat{p}(s) x(s)$ is $D_{H K}$ integrable on $[0, T]$ for all $x \in[y, z]$.

As a matter of fact, the proof of Lemma 3.1 follows exactly the lines of [5, Lemma 3.1], so we omit it here.

In what follows we recall a fixed point theorem for increasing mappings, which is an important tool for proving the existence theorem.

Let $E$ be an ordered Banach space, $K$ be a nonempty subset of $E$. The mapping $\mathcal{A}: K \rightarrow E$ is increasing if and only if $\mathcal{A} x \leq \mathcal{A} y$, whenever $x, y \in K$ and $x \leq y$.

Lemma 3.2 ([14, Theorem 3.1.3]) Let $y_{0}, z_{0} \in E$ with $y_{0}<z_{0}$ and $\mathcal{A}:\left[y_{0}, z_{0}\right] \rightarrow E$ be an increasing mapping satisfying $y_{0} \leq \mathcal{A} y_{0}, \mathcal{A} z_{0} \leq z_{0}$. If $\mathcal{A}\left[y_{0}, z_{0}\right]$ is relatively compact, then $\mathcal{A}$ has a maximal fixed point $x^{*}$ and a minimal fixed point $x_{*}$ in $\left[y_{0}, z_{0}\right]$. Moreover,

$$
x_{*}=\lim _{n \rightarrow \infty} y_{n}, \quad x^{*}=\lim _{n \rightarrow \infty} z_{n},
$$


where $y_{n}=\mathcal{A} y_{n-1}$ and $z_{n}=\mathcal{A} z_{n-1}(n=1,2,3, \ldots)$,

$$
y_{0} \leq y_{1} \leq \cdots \leq y_{n} \leq \cdots \leq x_{*} \leq x^{*} \leq \cdots \leq z_{n} \leq \cdots \leq z_{1} \leq z_{0} .
$$

We are now ready to give the main results.

Theorem 3.1 Let the functions $f$ and $h$ in (1.1) satisfy the assumptions $\left(\mathrm{D}_{1}\right)-\left(\mathrm{D}_{3}\right)$. Then the extremal solutions of PBVP (1.1) exist in the ordering interval $[y, z]$.

Proof Let

$$
g(t, x)=h(t, x)+p(t) x, \quad t \in[0, T], x \in[y, z],
$$

where $p(t)$ is given in $\left(D_{1}\right)$. Then the hypotheses $\left(D_{1}\right)-\left(D_{3}\right)$ imply, for all $x \in[y, z]$, that $g(\cdot, x(\cdot))$ is Lebesgue integrable and

$$
D y+c_{y}+p y \preceq f+g(\cdot, x) \preceq D z-c_{z}+p z \quad \text { on }[0, T] .
$$

Define a mapping $\mathcal{A}$ on $[y, z]$ by

$$
\begin{aligned}
\mathcal{A} x(t)= & e^{-P(t)}\left(D_{H K}\right) \int_{0}^{t} e^{P(s)}(f(s)+g(s, x(s))) d s \\
& +\frac{e^{-P(t)}}{e^{P(T)}-1}\left(D_{H K}\right) \int_{0}^{T} e^{P(s)}(f(s)+g(s, x(s))) d s, \quad t \in[0, T] .
\end{aligned}
$$

It follows from (3.7) that, for each $x \in[y, z]$,

$$
\begin{aligned}
& D(\mathscr{A} x)=f+g(\cdot, x)-p \mathscr{A} x \quad \text { on }[0, T], \\
& \mathcal{A} x(0)=\mathcal{A} x(T) .
\end{aligned}
$$

Let $w=\mathcal{A} y-y$. Then, by $\left(\mathrm{D}_{1}\right),(3.5)$ and (3.8), the inequalities

$$
D w+p w \succeq c_{y}
$$

and

$$
w(T)-w(0) \leq\left(D_{H K}\right) \int_{t}^{T} e^{P(s)-P(T)} c_{y}(s) d s+\left(D_{H K}\right) \int_{0}^{t} e^{P(s)} c_{y}(s) d s, \quad t \in[0, T]
$$

hold. It follows from (3.9) that

$$
e^{P(t)} w(t) \geq w(0)+\left(D_{H K}\right) \int_{0}^{t} e^{P(s)} c_{y}(s) d s, \quad t \in[0, T]
$$

Inequalities (3.10) and (3.11) imply, for each $t \in[0, T]$, that

$$
\begin{aligned}
w(0)+\left(D_{H K}\right) \int_{0}^{t} e^{P(s)} c_{y}(s) d s & \geq w(T)-\left(D_{H K}\right) \int_{t}^{T} e^{P(s)-P(T)} c_{y}(s) d s \\
& \geq e^{-P(T)} w(0)+\left(D_{H K}\right) \int_{0}^{t} e^{P(s)-P(T)} c_{y}(s) d s
\end{aligned}
$$


This result implies

$$
w(0)+\left(D_{H K}\right) \int_{0}^{t} e^{P(s)} c_{y}(s) d s \geq 0, \quad t \in[0, T] .
$$

In view of (3.11) and (3.12), we then have $w=\mathcal{A} y-y \geq 0$, i.e., $y \leq \mathcal{A} y$. We can similarly verify that $\mathcal{A} z \leq z$.

It follows from $\left(\mathrm{D}_{3}\right)$ and (3.5) that $g(t, \cdot)$ is nondecreasing on $[y, z]$ for all $t \in[0, T]$. Moreover, $\mathcal{A} x \in C[0, T]$ for each $x \in[y, z]$, whence (3.7) defines a nondecreasing mapping $\mathcal{A}:[y, z] \rightarrow[y, z]$.

We now only need to prove that $\mathscr{A}[y, z]$ is relatively compact.

The properties of $\mathcal{A}$ imply that

$$
y(t) \leq \mathcal{A} x(t) \leq z(t), \quad x \in[y, z], t \in[0, T]
$$

Since $y, z \in C[0, T]$ are bounded, there exists $N>0$ such that

$$
|\mathcal{A} x(t)|<N, \quad x \in[y, z], t \in[0, T] .
$$

This implies that $\mathcal{A}[y, z]$ is uniformly bounded.

Let $t_{1}, t_{2} \in[0, T]$. Then, by (3.7), for each $x \in[y, z]$,

$$
\begin{aligned}
\mathcal{A} x\left(t_{1}\right)-\mathcal{A} x\left(t_{2}\right)= & e^{-P\left(t_{1}\right)}\left(D_{H K}\right) \int_{t_{2}}^{t_{1}} e^{P(s)}(f(s)+g(s, x(s))) d s \\
& +\left(e^{P\left(t_{2}\right)-P\left(t_{1}\right)}-1\right) \mathcal{A} x\left(t_{2}\right) .
\end{aligned}
$$

Since $p \in H K$ and $p(t) \geq 0$ on $[0, T], P(t)=(H K) \int_{0}^{t} p(s) d s$ is continuous and so it is uniformly continuous on $[0, T]$. Then, for any $\varepsilon>0$, there exists $\delta>0$ such that

$$
\left|e^{P\left(t_{2}\right)-P\left(t_{1}\right)}-1\right| \leq \varepsilon, \quad \text { whenever } t_{1}, t_{2} \in[0, T] \text { and }\left|t_{2}-t_{1}\right| \leq \delta .
$$

Moreover, the continuity of $e^{P(t)}$ on $[0, T]$ implies that there exists $M>0$ such that

$$
\frac{1}{M}<e^{P(t)}<M, \quad t \in[0, T] .
$$

On the other hand, the monotonicity of $g(t, \cdot)$ on $[y, z]$ for all $t \in[0, T]$ and $(2.2)$ implies that

$$
\begin{aligned}
\left(D_{H K}\right) \int_{t_{2}}^{t_{1}} e^{P(s)}(f(s)+g(s, y(s))) d s & \leq\left(D_{H K}\right) \int_{t_{2}}^{t_{1}} e^{P(s)}(f(s)+g(s, x(s))) d s \\
& \leq\left(D_{H K}\right) \int_{t_{2}}^{t_{1}} e^{P(s)}(f(s)+g(s, z(s))) d s .
\end{aligned}
$$

This result, (3.13), (3.14), (3.15) and (3.16) imply that

$$
\begin{aligned}
& \left|\mathcal{A} x\left(t_{1}\right)-\mathcal{A} x\left(t_{2}\right)\right| \\
& \quad \leq M\left|\left(D_{H K}\right) \int_{t_{2}}^{t_{1}} e^{P(s)}(f(s)+g(s, x(s))) d s\right|+N \varepsilon
\end{aligned}
$$




$$
\begin{aligned}
\leq & M\left(\left|\left(D_{H K}\right) \int_{t_{2}}^{t_{1}} e^{P(s)}(f(s)+g(s, y(s))) d s\right|\right. \\
& \left.+\left|\left(D_{H K}\right) \int_{t_{2}}^{t_{1}} e^{P(s)}(f(s)+g(s, z(s))) d s\right|\right)+N \varepsilon .
\end{aligned}
$$

Since $e^{P(t)}(f(t)+g(t, y(t)))$ and $e^{P(t)}(f(t)+g(t, z(t)))$ are $D_{H K}$-integrable on $[0, T]$, the primitives of them are continuous and so they are uniformly continuous on $[0, T]$. Hence, by inequality (3.17), $\mathcal{A}[y, z]$ is equiuniformly continuous on $[0, T]$ for all $x \in[y, z]$. In view of the Ascoli-Arzelà theorem, $\mathcal{A}[y, z]$ is relatively compact. Thus, $\mathcal{A}$ satisfies the hypotheses of Lemma 3.2, whence $\mathcal{A}$ has the minimal fixed point $x_{*}$ and the maximal fixed point $x^{*}$. From the definitions of $g$ and $\mathcal{A}$ and Lemma 3.1 it follows that $x_{*}$ and $x^{*}$ are also solutions of PBVP (1.1) in $[y, z]$. Moreover, (3.3) and (3.4) hold with $y_{0}, z_{0}$ replaced by $y, z$.

If $x$ is any solution of PBVP (1.1) in $[y, z]$, then it is, by Lemma 3.1, a fixed point of $\mathcal{A}$, whence $x_{*} \leq x \leq x^{*}$. Thus $x_{*}$ and $x^{*}$ are the minimal and maximal solutions of PBVP (1.1) in $[y, z]$, respectively.

Remark 3.2 Let $p(t) \equiv M, c_{y}=M r_{y}$ and $c_{z}=M r_{z}$, where $M$ is a positive constant, $r_{y}=$ $(y(0)-y(T)) \frac{e^{P(T)}}{e^{P(T)}-1} \neq 0$ and $r_{z}=(z(T)-z(0)) \frac{e^{P(T)}}{e^{P(T)}-1} \neq 0$. Then $\left(\mathrm{D}_{1}\right)$ is reduced to

$\left(\mathrm{D}_{1}^{\prime}\right)$ There exist $y, z \in C[0, T], y \leq z$, such that $D y \preceq f+h(\cdot, y)-M r_{y}, D z \succeq f+h(\cdot, z)+M r_{z}$ on $[0, T], y(0)>y(T)$ and $z(0)<z(T)$.

If $c_{y}=c_{z}=0$ in $\left(\mathrm{D}_{1}\right)$, then we obtain

$\left(\mathrm{D}_{1}^{\prime \prime}\right)$ There exist $y, z \in C[0, T], y \leq z$, such that $D y \preceq f+h(\cdot, y)$ and $D z \succeq f+h(\cdot, z)$ on $[0, T]$, $y(0) \leq y(T)$ and $z(0) \geq z(T)$.

Further, the $D_{H K}$-integral includes the Lebesgue integral, and the distributional derivative contains the ordinary derivative. Thus, we can see that Theorem 3.1 is a proper generalization of [2, Theorem 2.1] if $f \equiv 0$ on $[0, T]$.

Corollary 3.1 Given a function $g$, assume that $g(t, \cdot)$ is nondecreasing on $C[0, T]$ for all $t \in[0, T], g(\cdot, x(\cdot))$ is Lebesgue integrable on $[0, T]$ for every fixed $x \in C[0, T]$, and there exist $g_{ \pm} \in D_{H K}$ such that, for all $x \in C[0, T]$,

$$
g_{-} \preceq g(\cdot, x) \preceq g_{+} \quad \text { on }[0, T] .
$$

If $p \in H K$ on $[0, T], p(t) \geq 0$ with $P(t)=(H K) \int_{0}^{t} p(s) d s$ nonzero at $t=T$ and $f$ is a distribution on $[0, T]$, then the PBVP

$$
D x+p(t) x=f(t)+g(t, x), \quad x(0)=x(T)
$$

has the extremal solutions.

Proof Let $y$ be a solution of the PBVP

$$
D y+p(t) y=f+g_{-}, \quad y(0)=y(T)
$$


and $z$ a solution of the PBVP

$$
D z+p(t) z=f+g_{+}, \quad z(0)=z(T) .
$$

According to Lemma 3.1, it is easy to see that $y, z$ are unique.

By choosing $h(t, x)=g(t, x)-p(t) x, t \in[0, T], x \in C[0, T]$, we have that the following two inequalities

$$
D y \preceq f+h(\cdot, y), \quad D z \succeq f+h(\cdot, z)
$$

hold if $y, z$ are given as above. Thus, the conditions $\left(D_{1}\right)-\left(D_{3}\right)$ are satisfied and, by Theorem 3.1, PBVP (3.19) has the extremal solutions $x_{*}$ and $x^{*}$ in $[y, z]$. Moreover, if $x$ is any solution of PBVP (3.19), it follows from Lemma 3.1 that for each $t \in[0, T]$,

$$
\begin{aligned}
x(t)= & e^{-P(t)}\left(D_{H K}\right) \int_{0}^{t} e^{P(s)}(f(s)+g(s, x(s))) d s \\
& +\frac{e^{-P(t)}}{e^{P(T)}-1}\left(D_{H K}\right) \int_{0}^{T} e^{P(s)}(f(s)+g(s, x(s))) d s .
\end{aligned}
$$

This result, (3.18), (3.20) and (3.21) imply that each solution of PBVP (3.19) belongs to $[y, z]$, whence $x_{*}$ and $x^{*}$ are the minimal and maximal solutions of PBVP (3.19).

We now give an example to illustrate the main results.

Example 3.1 Consider the PBVP given by

$$
D x+x=D r+\arctan x, \quad x(0)=x(2),
$$

where $D r$ is the distributional derivative of

$$
r(t)=\sum_{n=1}^{\infty} \frac{\sin n^{2} \pi t}{n^{2}} .
$$

Then PBVP (3.22) has extremal solutions.

Proof PBVP (3.22) can be regard as a PBVP of the form (3.19), where

$$
p(t) \equiv 1, \quad f=D r, \quad g(t, x)=\arctan x, \quad t \in[0,2], x \in C[0,2] .
$$

It is easy to see that $0 \leq p(t) \in H K$ and $(H K) \int_{0}^{2} p(s) d s=2>0$. Choose $g_{ \pm}(t)= \pm \frac{\pi}{2}$, then $g_{ \pm} \in D_{H K}$ and (3.18) holds. This result and $g(t, \cdot)$ is nondecreasing imply that the hypotheses of Corollary 3.1 are satisfied. The proof is therefore completed.

It is well known that the function $r(t)$ in (3.23) given by Riemann and proved by Hardy [15] is continuous but pointwise differentiable nowhere on $\mathbb{R}$. Then, by [5, Example 2.4], the distributional derivative $D r$ is neither $H K$-integrable nor Lebesgue integrable. Hence, [2, Theorem 2.1] is not applicable in this case, which implies that the main results in this paper are more general. 
The following result shows the dependence of the extremal solutions of PBVP (1.1) on $f$ and $h$.

Proposition 3.1 If the hypotheses of Theorem 3.1 hold, then the extremal solutions of PBVP (1.1) in $[y, z]$ are nondecreasing with respect to $f$ and $h$.

Proof Let $f, \hat{f}, h$ and $\hat{h}$ satisfy, for all $x \in C[0, T]$,

$$
f+h(\cdot, x) \preceq \hat{f}+\hat{h}(\cdot, x) \quad \text { on }[0, T] .
$$

Assume that the hypotheses of Theorem 3.1 hold for $f, h$ and $\hat{f}, \hat{h}$ with the same fixed functions $y, z, c_{y}, c_{z}$ and $p$. Let $x_{*}$ be the minimal solution of PBVP (1.1) in $[y, z], \hat{x}_{*}$ the minimal solution of the PBVP

$$
D x=\hat{f}(t)+\hat{h}(t, x), \quad x(0)=x(T)
$$

in $[y, z]$. Let $\mathcal{A}:[y, z] \rightarrow[y, z]$ be defined by (3.7), where $g$ is given by (3.5). By Lemma 3.1, one has

$$
\begin{aligned}
\hat{x}_{*}(t)= & e^{-P(t)}\left(D_{H K}\right) \int_{0}^{t} e^{P(s)}\left(\hat{f}(s)+\hat{g}\left(s, \hat{x}_{*}(s)\right)\right) d s \\
& +\frac{e^{-P(t)}}{e^{P(T)}-1}\left(D_{H K}\right) \int_{0}^{T} e^{P(s)}\left(\hat{f}(s)+\hat{g}\left(s, \hat{x}_{*}(s)\right)\right) d s, \quad t \in[0, T],
\end{aligned}
$$

where

$$
\hat{g}(t, x)=\hat{h}(t, x)+p(t) x, \quad t \in[0, T], x \in[y, z] .
$$

It follows from (3.5), (3.24) and (3.27) that for all $x \in[y, z]$,

$$
f+g(\cdot, x) \preceq \hat{f}+\hat{g}(\cdot, x) \quad \text { on }[0, T]
$$

whence (3.7) and (3.26) imply that $\mathcal{A} \hat{x}_{*} \leq \hat{x}_{*}$, yet Lemma 3.2 holds with $y_{0}=y, z_{0}=\hat{x}_{*}$. Indeed, by (3.3), $x_{*}$ is also the minimal fixed point of $\mathcal{A}$ in $\left[y, \hat{x}_{*}\right]$. This and (3.4) imply that $x_{*} \leq \hat{x}_{*}$.

Similarly, it is easy to verify that $x^{*} \leq \hat{x}^{*}$, where $x^{*}$ denotes the maximal solution of PBVP (1.1) in $[y, z]$ and $\hat{x}^{*}$ is the maximal solution of PBVP (3.25) in $[y, z]$.

\section{Competing interests}

The authors declare that they have no competing interests.

\section{Authors' contributions}

WL wrote the first draft and TA and GY corrected and improved the final version. All authors read and approved the final draft.

\section{Acknowledgements}

The authors would like to express their heartfelt appreciations to the expert referees for their helpful comments and suggestions. 


\section{References}

1. Lakshmikantham, $\mathrm{V}$, Leela, S: Existence and monotone method for periodic solutions of first-order differential equations. J. Math. Anal. Appl. 91(1), 237-243 (1983)

2. Lakshmikantham, $\mathrm{V}$, Leela, S: Remarks on first and second order periodic boundary value problems. Nonlinear Anal. 8(3), 281-287 (1984)

3. Bereanu, C, Mawhin, J: Existence and multiplicity results for periodic solutions of nonlinear difference equations. J. Differ. Equ. Appl. 12(7), 677-695 (2006)

4. Bereanu, C, Mawhin, J: Periodic solutions of first order nonlinear difference equations. Rend. Semin. Mat. (Torino) 65(1), 17-33 (2007)

5. Liu, W, Ye, GJ, Wang, Y, Zhou, XY: On periodic solutions for first order differential equations involving the distributional Henstock-Kurzweil integral. Bull. Aust. Math. Soc. 86, 327-338 (2012)

6. Lee, PY: Lanzhou Lecture on Henstock Integration. World Scientific, Singapore (1989)

7. Chew, TS, Lee, PY: The topology of the space of Denjoy integrable functions. Bull. Aust. Math. Soc. 42(3), 517-524 (1990)

8. Lee, TY: A full descriptive definition of the Henstock-Kurzweil integral in the Euclidean space. Proc. Lond. Math. Soc 87(3), 677-700 (2003)

9. Talvila, E: The distributional Denjoy integral. Real Anal. Exch. 33(1), 51-82 (2008)

10. Talvila, E: Convolutions with the continuous primitive integral. Abstr. Appl. Anal. 2009, Article ID 307404 (2009). doi:10.1155/2009/307404

11. Schwabik, Š, Ye, GJ: Topics in Banach Space Integration. World Scientific, Singapore (2005)

12. Lu, YP, Ye, GJ, Liu, W, Wang, Y: Existence of solutions of the wave equation involving the distributional Henstock-Kurzweil integral. Differ. Integral Equ. 24(11-12), 1063-1071 (2011)

13. Ang, DD, Schmitt, K, Vy, LK: A multidimensional analogue of the Denjoy-Perron-Henstock-Kurzweil integral. Bull. Belg Math. Soc. Simon Stevin 4, 355-371 (1997)

14. Guo, DJ, Cho, YJ, Zhu, J: Partial Ordering Methods in Nonlinear Problems. Nova Science Publishers, New York (2004)

15. Hardy, GH: Weierstrass's non-differentiable function. Trans. Am. Math. Soc. 17(3), 301-325 (1916)

10.1186/1687-2770-2014-54

Cite this article as: Liu et al.: On first-order periodic boundary value problems and distributional Henstock-Kurzweil integrals. Boundary Value Problems 2014, 2014:54

\section{Submit your manuscript to a SpringerOpen ${ }^{\circ}$ journal and benefit from:}

- Convenient online submission

- Rigorous peer review

- Immediate publication on acceptance

- Open access: articles freely available online

- High visibility within the field

- Retaining the copyright to your article 